\title{
Extramedullary transformation of chronic myeloid leukaemia with a unique chromosome pattern
}

\author{
NG FLANAGAN,${ }^{*}$ CJ HARRISON,$\dagger$ DS HARRY, ${ }^{*}$ JC RIDWAY* \\ From the ${ }^{*}$ Departments of Haematology, Histopathology, and Immunology, Victoria Hospital, Blackpool, \\ and the $†$ Department of Cell Biology, Paterson Laboratories, Christie Hospital and Holt Radium Institute, \\ Manchester
}

SUMMARY A case of extramedullary myeloblastic transformation of chronic myeloid leukaemia is reported. This was characterised by an unusual clinical and haematological course and showed a unique cytogenetic pattern.

\section{Case report}

A 32 year old engineer was referred for an orthopaedic opinion having suffered from episodic aching in both knees for over three years. These episodes usually lasted three to four days and often interfered with walking. He had attributed the symptoms to trauma at regular rugby sessions and reported to his doctor only when they became more frequent. He otherwise felt well and had no relevant medical history. There was a strong family history of gout.

On examination he had slight tenderness on the medial aspect of both patellae, but there were no other significant findings. A diagnosis of retropatellar pain syndrome was made and was supported by $x$ ray findings of mild osteoarthritic changes in both knees. A biochemical profile was requested to exclude gout. A blood count was not taken at this time. The only biochemical abnormality was a considerably increased lactic dehydrogenase value, and in view of this he was referred to the haematology clinic.

He deteriorated quickly, however, and was admitted to hospital before his outpatient appointment. On admission he was feverish, restless, and distressed. He had gross cervical, axillary, and inguinal lymphadenopathy, which was slighly tender. His abdomen was tender in all areas with some guarding; the spleen was enlarged to $13 \mathrm{~cm}$ below the costal margin, the liver to $4 \mathrm{~cm}$. Results of initial haematological investigations were as follows: haemoglobin concentration $8.6 \mathrm{~g} / \mathrm{dl}$, total white cell count $151 \times 10^{9} / 1$, and platelet count $102 \times 10^{9} / 1$. A peripheral blood film showed $11 \%$ myeloblasts in an

Accepted for publication 28 February 1985 otherwise typical picture of chronic myeloid leukaemia. The marrow smear supported this diagnosis with an myeloid/erythroid ratio of 32:1. The leucocyte alkaline phosphatase score was 1 , and $\alpha$-naphthyl acetate and butyrate esterases were negative in the blasts. The serum $B_{12}$ concentration was greater than $1400 \mathrm{ng} / \mathrm{l}$, and the biochemical profile again showed a considerably raised lactate dehydrogenase value as the only abnormality. Cell surface markers showed a normal pattern on the lymphocytes with the blast cells negative for cellular terminal deoxynucleotidyl transferase (TdT). The result of a Paul-Bunnell was negative. Enlarged nodes were seen at the left hilum on chest $x$ ray examination. Chromosome analysis was carried out on the peripheral blood; unstimulated cultures were examined. Twenty five G-banded cells showed the following karyotype (Fig. 1):

$46, X Y, t(9 ; 22)(q 34 ; q 11)$ in 17 cells; $46, X Y, t(9 ; 22)$ (q34; 111$),-18$ in two cells; $46, X Y, t(9 ; 22)$ ( $\mathrm{q} 34 ; \mathrm{q} 11)$, direct insertion $(3 ; 13)$ (q21;q12q34) in six cells.

A sample of blood stimulated with phytohaemagglutinin showed 10 cells with a 46,XY, $t(9 ; 22)$ (q34;q11) karyotype.

A firm diagnosis of chronic myeloid leukaemia was made. The clinical features suggested that this might be in the accelerated phase, yet only $11 \%$ blasts were found in the peripheral blood. He was given oral busulphan and 6-thioguanine daily, and after 10 days he had shown a gratifying response with lymph nodes barely palpable and a steady fall in his total white cell count. When seen as an outpatient after three weeks he felt well, splenomegaly had regressed to $8 \mathrm{~cm}$, and the marrow smear was virtually within normal limits. His peripheral blood picture was normal. The chemotherapy was reduced 


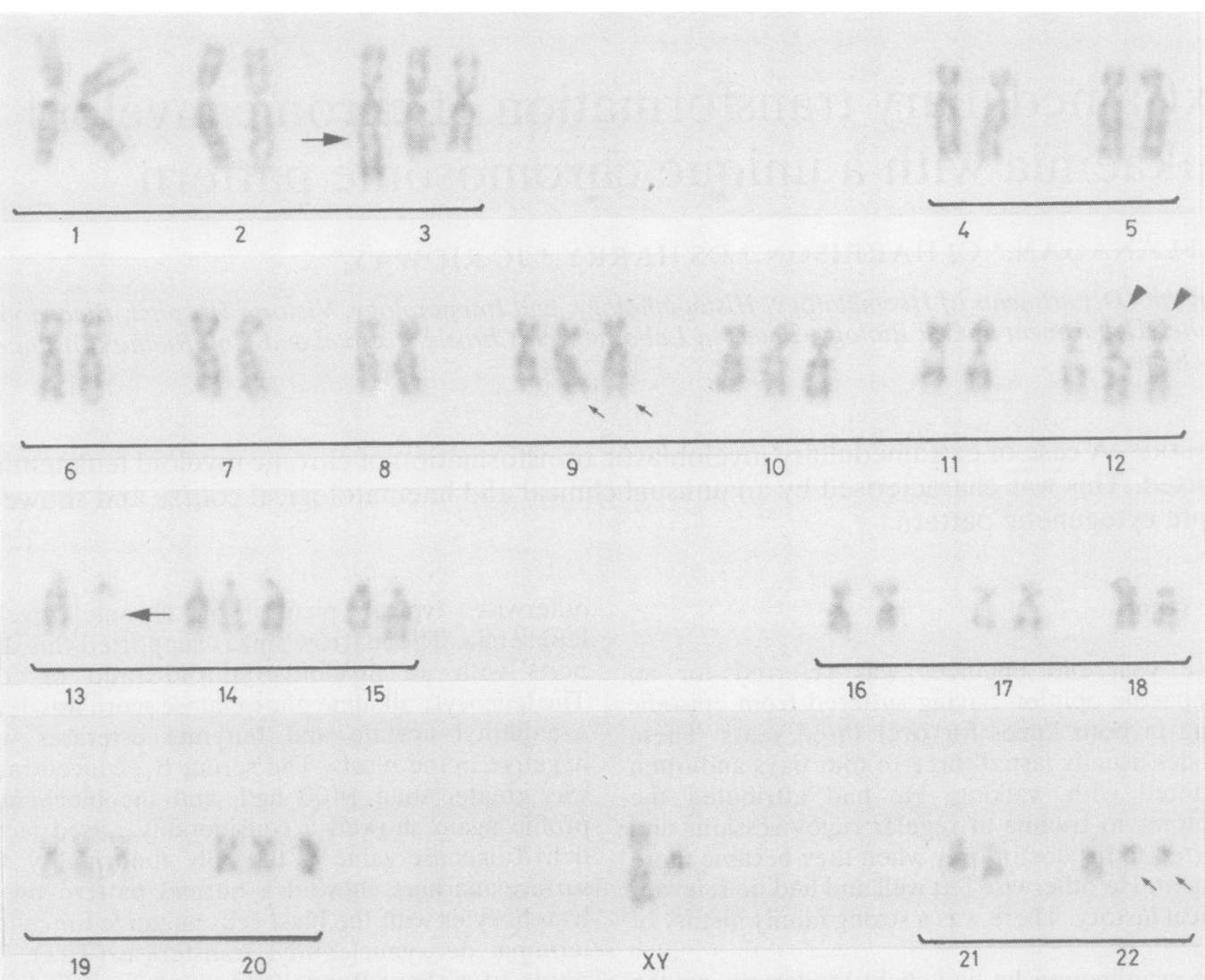

Fig. 1 Karyotype from bone marrow sample in blastic crisis. Additional chromosomes 3, 10, 12, 14, 19 and 20 are present. The dir ins $(3 ; 13)(q 21 ; q 12 q 34)$ is shown (arrows). The original and an additional $t(9 ; 22)$ $(q 34 ; q 11)$ are indicated (small arrows). Two 12p + chromosomes are also shown (arrowheads).

to three days a week.

He remained well for about four weeks but then reported unexpectedly with a two day history of dysphagia; he again had greatly enlarged cervical lymph nodes. Node biopsy at this time showed features of a poorly differentiated lymphocytic lymphoma, which did not penetrate the capsule (Fig. 2). In the node imprints, however, the cells had myeloid features with two or three conspicuous nucleoli, though no granules were present, and these cells were negative for immunoglobulins, chloracetate esterase, and muramidase. There were no accompanying eosinophilic or basophilic myeloid cells. Electron microscopy did not give specific results. A marrow sample at this time was consistent with chronic myeloid leukaemia responding to treatment, and the cellular TdT on this sample was negative. Within 10 days he was showing signs of tracheal compression and had gross generalised lym- phadenopathy. He was transferred for urgent radiotherapy to the Christie Hospital, Manchester. At this time chromosome analysis on unstimulated cells extracted from the node showed: 46,XY $\mathrm{t}(9 ; 22)(\mathrm{q} 34 ; \mathrm{q} 11)$ dir ins $(3 ; 13)(\mathrm{q} 21 ; \mathrm{q} 12 \mathrm{q} 34)$ in all cells examined ( 20 cells). A repeat marrow smear was normal. The cervical nodes regressed quickly o after initial radiotherapy. He was seen at weekly intervals as an outpatient; at first he received low $\triangle$ dose whole body irradiation but soon changed to $\omega$ lower body irradiation only because of increasing adenopathy and pain in this area. His blood picture 0 was unaltered for six weeks; a further marrow smear $\mathbb{D}$ showed typical features of acute myeloid leukaemia $\stackrel{?}{?}$ with $90 \%$ blasts. On his return to this department ${ }^{\circ}$ his total white cell count was $101 \times 10^{9} / 1$ with $96 \%$ blasts. Combined esterase staining was negative. $\cong$ Blast cells were positive for HLA-Dr and negative $\mathbb{Q}$ for TdT and common acute lymphoblastic 


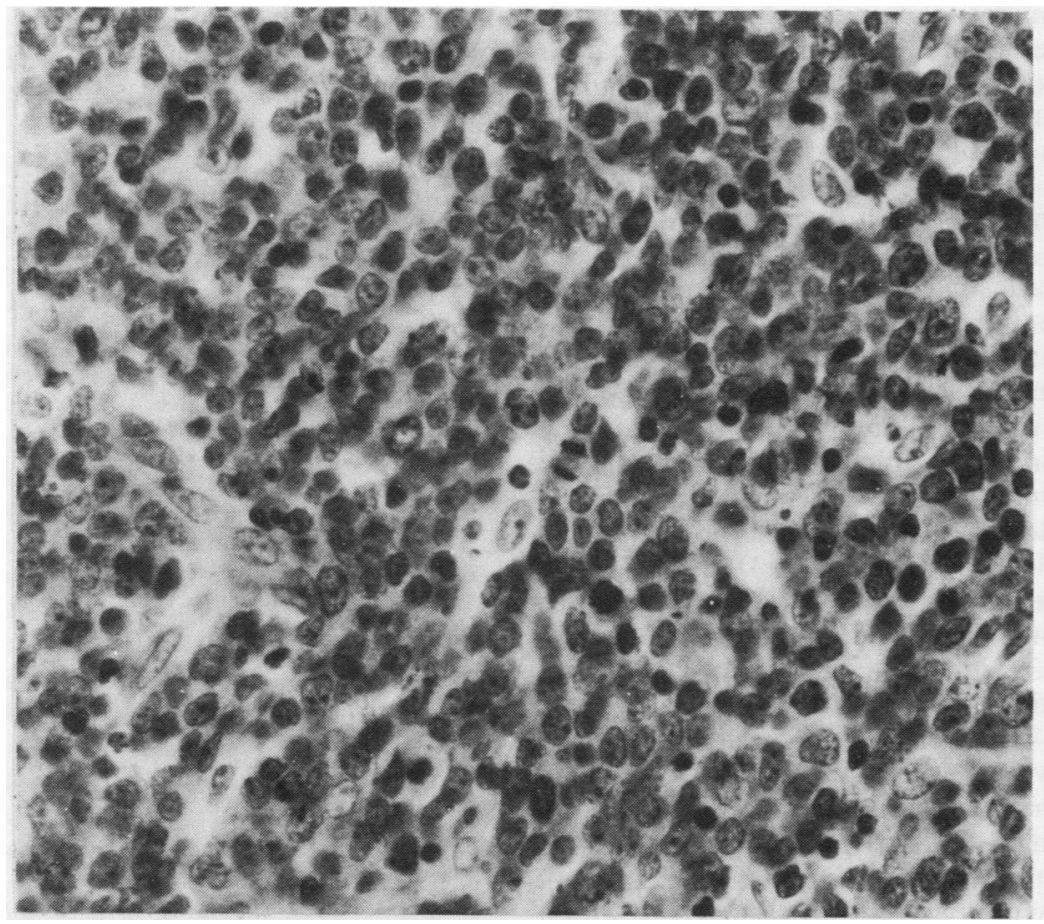

Fig. 2 Cellular detail of lymph node. (Haematoxylin and eosin. $\times 1000$ (approx).

leukaemia antigen. He showed a modest response to intensive chemotherapy, but then he steadily deteriorated and died almost exactly six months after initial presentation. Chromosome studies on a marrow sample when he entered the final blastic crisis showed additional changes in a large percentage of cells as follows:

$54, \mathrm{XY}, \mathrm{t}(9 ; 22)(\mathrm{q} 34 ; \mathrm{q} 11),+\mathrm{t}(9 ; 22)(\mathrm{q} 34 ; \mathrm{q} 11),+3$, +9 , $+10,+12,+14,+19$, +20 , dir ins $(3 ; 13)$ (q21;q21q34), 12p+, 12p+ in 16 cells;

46,XY, $t(9 ; 22)(q 34 ; q 11)$, dir ins $(3 ; 13)$ ( $\mathrm{q} 21 ; \mathrm{q} 21 \mathrm{q} 34)$ in 13 cells. No cells were seen without the $(3 ; 13)$ chromosome at this stage.

At necropsy the spleen was considerably enlarged (weight $1820 \mathrm{~g}$ ), but only moderate lymphadenopathy was found showing the same features as the earlier lymph node biopsy. Severe haemorrhagic pulmonary oedema appeared to be a main cause of death.

\section{Discussion}

Although lymphadenopathy is an unusual feature in chronic myeloid leukaemia, it is well documented..$^{1-3}$ In fact, the lymph nodes have been the most consistent site of extramedullary transformation. Because of the primitive cellular infiltration into the lymph nodes, the possibility of a coexistent lymphoma with chronic myeloid leukaemia must be considered; indeed several such cases have been reported. ${ }^{24}$ Joseph et al ${ }^{5}$ and Lazlo and Grode ${ }^{6}$ suspected the primitive cells were undifferentiated myeloid cells and subsequent studies ${ }^{4}$ staining the sections with the Giemsa and naphthol AS-D chloroacetate technique supported this. In our patient the failure of the infiltrate to penetrate the node capsule did not favour lymphoma, and the node imprint showed a few cells with definite myeloid characteristics.

Our patient showed several unusual features. The lymphadenopathy was gross, appearing and regressing (after treatment) with remarkable rapidity. Two weeks before his first admission he had been examined by an experienced clinician; who found no signs of splenomegaly or lymphadenopathy. At that time he was still playing rugby. On admission, however, he was desperately ill and showed convincing evidence of chronic myeloid leukaemia. His initial response to our usual oral chemotherapy for this condition was remarkably good. In our experience it is most unusual for the marrow in chronic phase chronic myeloid leukaemia to return to normal so quickly on this regimen, although the peripheral blood picture does rapidly improve. Repeated nor- 
mal marrow samples taken during his radiotherapy, when chemotherapy had been discontinued, raised considerable doubts about the original diagnosis. These were dispelled after a review of all available material and in the light of the subsequent progress of the disease. Cell surface markers failed to show evidence of lymphoid transformation in the samples examined, but the lymph node biopsy sample was not available for testing. HLA-Dr was the only positive marker identified on the blast cells and this was present only in the last sample tested. The cytogenetic studies were particularly interesting. In the first sample taken every cell in his unstimulated and stimulated peripheral blood possessed a $\mathbf{P h}^{1}$ chromosome, indicating an advanced stage of the disease. In addition, a direct insertion affecting chromosomes 3 and 13 (dir ins $(3 ; 13)$ (q21;q12q34) was found in a percentage of his cells. Numerous unusual chromosome abnormalities affecting chromosomes 3 and 13 have been described in chronic myeloid leukaemia, ${ }^{7}$ but this particular rearrangement has not been previously described. It is apparently a cytogenetic change subsequent to the $\mathrm{Ph}^{1}$ chromosome as it was found in only some of the cells at this stage. In the lymph node biopsy this secondary change was found in all cells examined. It was also present in all cells in blastic crisis, with a high proportion of cells showing numerous additional abnormalities. These types of changes are highly characteristic of chronic myeloid leukaemia blastic crisis, occurring in $70 \%$ of cases. ${ }^{8}$ Additional $\mathrm{Ph}^{1}$ chromosomes and chromosome 8 are the most common changes, and were also shown by this patient. A review of other cases of extramedullary myeloblastic transformation of chronic myeloid leukaemia strongly supports the view that this feature is a grave prognostic sign:

We thank Drs Chang and Gupta of the Christie Hospital, and Miss E Jones for secretarial help.

\section{References}

' Woodson DL, Bennett DE, Sears DA. Extramedullary myeloblastic transformation of chronic myelocytic leukaemia. Arch Intern Med 1974;134:523-6.

${ }^{2}$ Talvalkar GV, Srinivasan U, Advani SH, Khare AG. Extramedullary blast crisis in chronic myeloid leukaemia simulating lymphoma. Indian J Cancer 1977;14:272-7.

${ }^{3}$ Efrati P, Nir E, Berrebi A, Yaari A, Rappaport S, Resnitzky P. Myeloblastic metamorphosis in lymph node in a case of chronic myeloid leukaemia. A clinical morphological ultrastructural and cytogenetic study. Haematologica 1982;67:3344.

${ }^{4}$ Garfinkel LS, Bennett DE. Extramedullary myeloblastic transformation in chronic myelocytic leukaemia simulating a coexistent malignant lymphoma. Am J Clin Pathol 1969; 51:638-45.

s Joseph RR, Zarafonetis CJO, Durant JR. "Lymphoma" in chronic granulocytic leukaemia. Am J Med Sci 1966; 251:417-27.

- Lazlo J, Grode HE. Granulocytic leukaemia and reticulum cell sarcoma. Cancer 1967; 20:545-51.

${ }^{7}$ Mitelman F. Catalogue of chromosome abberations in cancer. Cytogenet Cell Genet 1981;36:1-2, 1-515.

${ }^{8}$ Sandberg AA, ed. Chromosomal findings in the blastic phase of CML. The chromosomes in human cancer and leukaemia. New York: Elsevier, 1980:235-49.

Requests for reprints to: Dr NG Flanagan, Department of Haematology, Victoria Hospital, Blackpool, England. 